\title{
In Vivo Evidence of Increased nNOS Activity in Acute MPTP Neurotoxicity: A Functional Pharmacological MRI Study
}

\author{
Tiing Yee Siow, ${ }^{1,2}$ Chiao-Chi V. Chen, ${ }^{1}$ Nina Wan, ${ }^{3}$ Kai-Ping N. Chow, ${ }^{4}$ and Chen Chang \\ ${ }^{1}$ Institute of Biomedical Sciences, Academia Sinica, 128 Section 2, Academia Road, Nankang, Taipei 11529, Taiwan \\ ${ }^{2}$ Department of Medical Imaging and Intervention, Chang-Gung Memorial Hospital, \\ Chang-Gung University College of Medicine, Taoyuan 33302, Taiwan \\ ${ }^{3}$ School of Nursing, Queen's University, Kingston, ON, Canada K7L 3N6 \\ ${ }^{4}$ Department of Microbiology and Immunology, Chang-Gung University, Taoyuan 33302, Taiwan
}

Correspondence should be addressed to Chen Chang; bmcchen@ibms.sinica.edu.tw

Received 13 January 2013; Revised 21 July 2013; Accepted 1 August 2013

Academic Editor: Tomio Inoue

Copyright (C) 2013 Tiing Yee Siow et al. This is an open access article distributed under the Creative Commons Attribution License, which permits unrestricted use, distribution, and reproduction in any medium, provided the original work is properly cited.

1-Methyl-4-phenyl-1,2,3,6-tetrahydropyridine (MPTP) is a neurotoxin commonly used to produce an animal model of Parkinson's disease. Previous studies have suggested a critical role for neuronal nitric oxide (NO) synthase- (nNOS-) derived NO in the pathogenesis of MPTP. However, NO activity is difficult to assess in vivo due to its extremely short biological half-life, and so in vivo evidence of NO involvement in MPTP neurotoxicity remains scarce. In the present study, we utilized flow-sensitive alternating inversion recovery sequences, in vivo localized proton magnetic resonance spectroscopy, and diffusion-weighted imaging to, respectively, assess the hemodynamics, metabolism, and cytotoxicity induced by MPTP. The role of NO in MPTP toxicity was clarified further by administering a selective nNOS inhibitor, 7-nitroindazole (7-NI), intraperitoneally to some of the experimental animals prior to MPTP challenge. The transient increase in cerebral blood flow $(\mathrm{CBF})$ in the cortex and striatum induced by systemic injection of MPTP was completely prevented by pretreatment with 7-NI. We provide the first in vivo evidence of increased nNOS activity in acute MPTP-induced neurotoxicity. Although the observed CBF change may be independent of the toxicogenesis of MPTP, this transient hyperperfusion state may serve as an early indicator of neuroinflammation.

\section{Introduction}

Parkinson's disease $(\mathrm{PD})$ is a neurodegenerative disorder that is caused by the progressive loss of dopaminergic (DAergic) neurons in the substantia nigra pars compacta $(\mathrm{SNpc})$. The cardinal manifestations of this debilitating disease include muscle rigidity, uncontrolled tremor, and bradykinesia. Much of the insight into PD has come from the animal model, in which the condition is induced by administration of the toxin 1-methyl-4-phenyl-1,2,3,6-tetrahydropyridine (MPTP), which faithfully reproduces the pathological hallmarks of PD. MPTP is initially converted to its toxic metabolic form, 1-methyl-4-phenylpyridinium ion $\left(\mathrm{MPP}^{+}\right)$, in vivo by monoamine oxidase- (MAO-)B (MAO-B) [1]. $\mathrm{MPP}^{+}$subsequently accumulates in DAergic neurons through high-affinity dopamine transporters [2]. Once inside neurons, $\mathrm{MPP}^{+}$ disrupts oxidative phosphorylation by inhibiting mitochondrial complex I of the electron transport chain [3-8]. It is hypothesized that interference with the cellular respiratory machinery leads to rapid depletion of adenosine triphosphate (ATP) and eventually cell death. However, it appears that complex I activity requires reduction of more than $70 \%$ to cause significant energy depletion in nonsynaptic brain mitochondria [9] and an in vivo study has shown that MPTP causes only a transient $20 \%$ reduction in ATP level in the mouse striatum and midbrain [10]. Together these data argue that ATP deficit is the sole factor underlying MPTP-induced neuron loss.

In addition to the ATP-depletion hypothesis, it has been postulated that increased production of nitric oxide (NO) also contributes to MPTP-induced neurotoxicity [11-15]. The impaired oxidative phosphorylation after administrating MPTP causes activation of $N$-methyl-D-aspartate receptors with subsequent increase in the intracellular $\mathrm{Ca}^{2+}$ concentration. This leads to the activation of neuronal NO synthase 
(nNOS), which is a calmodulin-dependent enzyme [16]. The subsequently produced NO combines with superoxide to form the free radical peroxynitrite [17], which in turn degenerates into a more noxious hydroxyl radical to cause cell injury. Nevertheless, $\mathrm{NO}$ activity is difficult to assess in vivo due to its extremely short biological half-life of only a few seconds [18]. In vivo evidence of NO involvement in MPTP neurotoxicity remains scarce.

As well as playing a part in neuroinflammation, NO is known to play a pivotal role in the regulation of vascular tone $[19,20]$. The central effect of NO in hemodynamic homeostasis provides a rationale for the present study, which examined the role of NO in the MPTP-induced neurotoxic cascade by monitoring alterations in CBF.

Over the past few decades, magnetic resonance imaging (MRI) has evolved into a powerful imaging modality that offers functional imaging in addition to anatomical information. Flow-sensitive alternating inversion recovery (FAIR) [21], a commonly used magnetic-resonance-based perfusion imaging technique, utilizes tissue water as an endogenous contrast agent to obtain tissue perfusion information. In addition to FAIR, functional imaging modalities such as diffusion-weighted imaging (DWI) and magnetic resonance spectroscopy (MRS) could provide useful information on cytotoxicity and metabolic changes. The noninvasiveness of these techniques enables repeated in vivo measurements with high temporal and spatial resolutions.

Combining these MRI techniques with pharmacological inquires, termed pharmacological MRI (phMRI) [22-24], has provided a platform for investigating drug effects in vivo. The present study used phMRI to investigate the acute effects of MPTP on the rodent central nervous system (CNS). FAIR, DWI, and in vivo localized proton magnetic resonance spectroscopy ( $\left.{ }^{1} \mathrm{H}-\mathrm{MRS}\right)$ were used to, respectively, assess MPTP-induced hemodynamic perturbations, cytotoxicity, and metabolic changes. To further clarify the role of NO in MPTP toxicity, a selective nNOS inhibitor, 7-nitroindazole (7NI) [25], was administered intraperitoneally (i.p.) to experimental animals prior to an MPTP challenge.

\section{Materials and Methods}

2.1. Animal Preparations. All experimental procedures were approved by the Institute of Animal Care and Utilization Committee at Academia Sinica, Taipei, Taiwan. Male Sprague-Dawley rats (4-5 months old) weighing 450-550 g were anesthetized i.p. with a mixture of urethane $(800 \mathrm{mg} / \mathrm{kg}$; Sigma, MO, USA) in normal saline and $\alpha$-chloralose (40 mg/kg, Sigma) in polyethylene glycol (Merck, Darmstadt, Germany). Each rat was placed in the prone position and fitted with a custom-designed head-holder. The rats were set up as described previously [26]. Briefly, one femoral vein was cannulated with PE-50 tubing for drug/test solution administration, and an endotracheal tube (PE-280) was inserted for artificial ventilation with an animal ventilator (Model 683, Harvard Instruments, South Natick, MA, USA). The expiratory $\mathrm{CO}_{2}$ concentration, which was monitored with the aid of a capnograph (Normocap 200, Datex, Helsinki, Finland), was maintained at $3.5-4.5 \%$ by adjusting the tidal volume and ventilation rate. An intravenous (i.v.) injection of a muscle relaxant, gallamine (Sigma), was used to prevent spontaneous ventilation and movement during the imageacquisition period. The initial dose of gallamine was $12 \mathrm{mgs}$ and the maintenance dosage was $6 \mathrm{mg} / \mathrm{h}$. Body temperature was detected by an optical fiber thermoprobe (Model SFF-5, Luxtron, Santa Clara, CA, USA) connected to a Fluoroptic thermometer (Model 790, Luxtron) and was maintained at $37^{\circ} \mathrm{C}$ by a ceramic heater (Model TH-8105, Tashin, Taipei, Taiwan) throughout the MRI measurements.

The rats were divided into three groups, with six rats in each group. In the first group, the rats received a single, i.v. injection of MPTP (15 mg/kg, Sigma), while the age-matched control group received an i.v. injection of normal saline. The third group of rats received a single dose of $7-\mathrm{NI}(50 \mathrm{mg} / \mathrm{kg}$ i.p., Sigma) $30 \mathrm{~min}$ prior to the i.v. administration of $15 \mathrm{mg} / \mathrm{kg}$ MPTP. As shown previously [27], maximal NOS inhibition in the rat brain is manifested within 30 min following the injection of 7-NI i.p.

2.2. MRI Protocols. All magnetic resonance experiments were performed on a 4.7-T Biospec 47/40 spectrometer with an active shielding gradient $(5.6 \mathrm{G} / \mathrm{cm}$ in $500 \mu \mathrm{s})$. A $20 \mathrm{~cm}$ birdcage coil was used for radiofrequency (RF) excitation, and $2 \mathrm{~cm}$ diameter surface coil was used for signal reception.

Conventional DWI was employed using a pulsedgradient spin-echo diffusion method, with a repetition time (TR) of $2000 \mathrm{~ms}$, an echo time (TE) of $59 \mathrm{~ms}$, a gradient pulse duration of $20 \mathrm{~ms}$, a time interval between diffusion gradient pulses of $27 \mathrm{~ms}$, and a $b$ value of $1300 \mathrm{~s} / \mathrm{mm}^{2}$. Images were obtained using a $5 \mathrm{~cm}$ field of view (FOV), a slice thickness of $2 \mathrm{~mm}$, a $256 \times 128$ matrix size that was zero filled to $256 \times 256$, and a total imaging time of $4 \mathrm{~min} 17 \mathrm{~s}$. The diffusion-sensitive gradients were applied in the read $(x)$ direction before and after the refocusing pulse. Hermite-shaped RF pulses with durations of 3 and $1.86 \mathrm{~ms}$ were used for the excitation and refocusing pulses, respectively.

The FAIR experiment was implemented with inversion recovery fast spin-echo (IR-FSE) sequences with and without a slice-selective gradient during an inversion pulse. Sliceselective IR-FSE (ssIR-FSE) and non-slice-selective IR-FSE (nsIR-FSE) images were collected using a TR of $3 \mathrm{~s}$, a TE of $20 \mathrm{~ms}$, and an effective TE of $50 \mathrm{~ms}$ with an echo train length of 4 , a slice thickness of $2 \mathrm{~mm}$, an FOV of $4 \mathrm{~cm}$, an inversion time $(\mathrm{TI})$ of $1.5 \mathrm{~s}$, and a matrix size of $256 \times 128$. A slab thickness of $5 \mathrm{~mm}$ was inverted for the ssIR-FSE images and a hyperbolic secant pulse was used for inversion with a pulse length of $8 \mathrm{~ms}$. The $T_{1}$ was measured from nsIR-FSE with TI values of $0.5,0.9,1.1,1.3,1.5$, and $1.9 \mathrm{~s}$.

A point-resolved spectroscopy (PRESS) sequence was used for localized spectroscopy with the following parameters: $5 \times 5 \times 5 \mathrm{~mm}^{3}$ voxel located at the striatal region, spectral width $=4000 \mathrm{~Hz}, \mathrm{TR}=2 \mathrm{~s}, \mathrm{TE}=136 \mathrm{~ms}$, number of average $=$ 256 , and total scanning time $=8 \mathrm{~min} 32 \mathrm{~s}$. Water suppression was achieved by chemical-shift-selective saturation, whereby three consecutive Hermite-shaped RF pulses, each of $15 \mathrm{~ms}$ duration, are applied followed by spoiling gradients preceding the PRESS sequence. Spectral assignments of the 
resonance lines in vivo were based on the results from in vitro ${ }^{1} \mathrm{H}-\mathrm{MRS}$.

2.3. Data Analysis. All data were processed using commercially available image-analysis software MRVision (MRVision Co., Menlo Park, CA, USA). The $T_{1}$ maps were produced using a nonlinear, three-parameter fitting procedure on a pixel-by-pixel basis. The FAIR images were generated by the subtraction of nsIR-FSE images from their corresponding ssIR-FSE images. The resulting images $(\Delta M)$ were used to generate $\mathrm{CBF}$ maps according to the following:

$$
f=\frac{\lambda \cdot \Delta M}{2 M_{0} \mathrm{TI} \exp \left(-\mathrm{TI} / T_{1}\right)},
$$

where $\lambda$ is the tissue-blood partition coefficient $(0.9 \mathrm{~mL} / \mathrm{g})$ [28], $M_{0}$ is the thermal equilibrium magnetization, and $f$ is the calculated CBF (expressed as $\mathrm{mL} / \mathrm{min} / 100 \mathrm{~g}$ of tissue, or $\mathrm{mL} / \mathrm{min} / 100 \mathrm{~g}$ ). The $M_{0}$ maps were calculated based on the $T_{1}$ maps and nsIR-FSE images using the following:

$$
M_{\mathrm{ns}}(\mathrm{TI})=M_{0}\left(1-2 \exp \left(\frac{\mathrm{TI}}{T_{1}}\right)\right),
$$

where $M_{\mathrm{ns}}$ is the magnetization in nonselective inversion.

Two regions of interest (ROIs) were analyzed in all cases: the entire cerebral cortex and the striatum. The average CBF was calculated within each ROI. All results are expressed as mean $\pm \mathrm{SD}$ values. Student's $t$-test was used for statistical evaluations, with the level of statistical significance set at $P<$ 0.05 .

\section{Results}

Administration of MPTP did not significantly change either the signal intensity or metabolite concentrations on DWI and in vivo ${ }^{1} \mathrm{H}$-MRS, respectively, throughout the $6 \mathrm{~h}$ experimental period (data not shown). However, FAIR revealed significant alterations in regional CBF. Figure 1 shows representative temporal CBF profiles from an MPTP-treated rat, a 7-NIpretreated and MPTP-treated rat, and a saline-treated rat. The basal CBFs in the cortex and striatum were $109 \pm 22$ and $102 \pm 17 \mathrm{~mL} / \mathrm{min} / 100 \mathrm{~g}$, respectively; these values are consistent with those reported previously [21,29]. There were no significant changes in $\mathrm{CBF}$ in brain region over time in the control rats injected with saline alone. However, in rats treated with MPTP alone, there were progressive elevations of CBFs in both the cortex and striatum. In both regions, the changes in CBF became significant at $29 \mathrm{~min}$ post-MPTP injection $(P<0.05$ for the striatum and $P<0.001$ for the cortex). The CBF was significantly higher in the cortex than in the striatum until 63 min after MPTP injection $(P<0.05)$.

As shown in Figure 2, the cortical CBF of MPTP-injected rats was significantly increased at $29 \mathrm{~min}$ after injection $(267 \pm 51 \mathrm{~mL} / \mathrm{min} / 100 \mathrm{~g}, P<0.001)$ reached its peak at $63 \mathrm{~min}(329 \pm 27 \mathrm{~mL} / \mathrm{min} / 100 \mathrm{~g}, P<0.001)$ but subsequently returned gradually to the control level at $165 \mathrm{~min}$ $(147 \pm 51 \mathrm{~mL} / \mathrm{min} / 100 \mathrm{~g})$, where it remained until the end of the experiment at $369 \mathrm{~min}$ after injection. The CBF increased less in the striatum than in the cortex at $29 \mathrm{~min}$

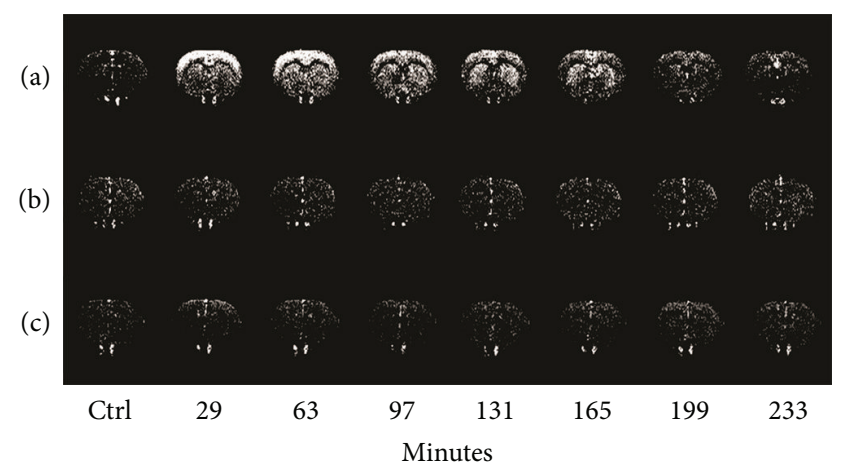

FIgURE 1: Temporal FAIR images. Representative temporal FAIR images from (a) an MPTP-treated rat, (b) a 7-NI-pretreated and MPTP-treated rat, and (c) a saline-treated (control) rat at baseline (ctrl) and various times postinjection. Progressive elevations of CBF were observed in the cortex and striatum of rats treated with MPTP alone. These elevations were prevented by pretreatment with 7-NI. There were essentially no changes in CBF in either brain region over time in the control rats.

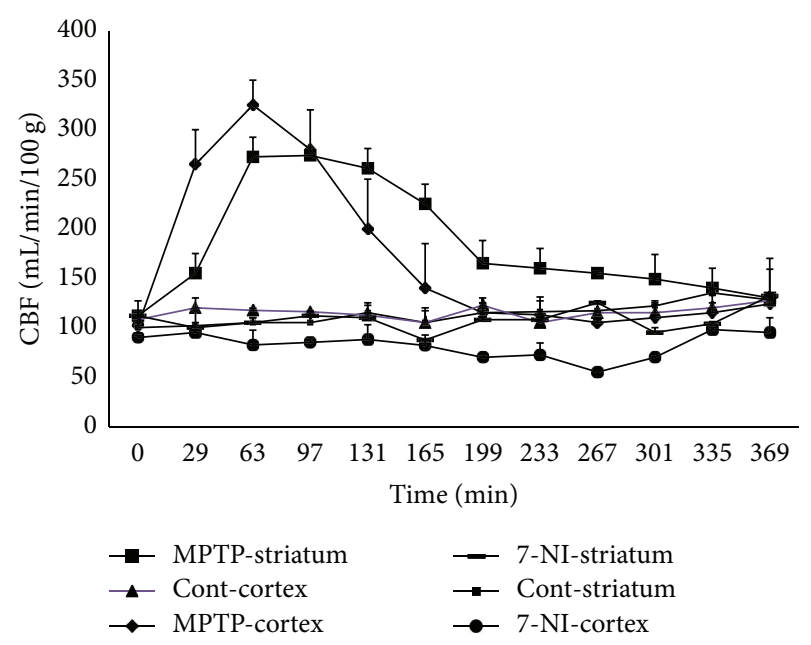

Figure 2: CBF changes over time. Temporal changes in CBF in the cortical and striatal regions of saline-treated (control (Cont)) and MPTP-treated rats. Pretreatment with 7-NI prevented the elevation of CBF induced by MPTP. Data are mean and SD values.

$(158 \pm 46 \mathrm{~mL} / \mathrm{min} / 100 \mathrm{~g}, P<0.05)$ and then reached its maximum at $97 \mathrm{~min}$ after injection $(273 \pm 42 \mathrm{~mL} / \mathrm{min} / 100 \mathrm{~g}$, $P<0.001$ ) but subsequently returned gradually to the control level at $335 \mathrm{~min}(140 \pm 21 \mathrm{~mL} / \mathrm{min} / 100 \mathrm{~g})$. The increase in CBF lasted longer in the striatum than in the cortex.

Pretreatment with 7-NI did not change basal striatal CBF, but it significantly attenuated the basal cortical CBF before MPTP treatment $(P<0.01)$. 7-NI completely blocked the MPTP-induced increase in CBF over time in both the cerebral cortex and the striatum. There was no significant MPTPinduced change in CBF in either the cerebral cortex or the striatum throughout the $6 \mathrm{~h}$ experimental period following pretreatment with 7-NI. 


\section{Discussion}

A possible role of $\mathrm{NO}$ in the pathogenic mechanism underlying the actions of MPTP has received considerable attention. There are several lines of evidence that implicated that neuronally derived NO at least partly mediates MPTP-induced $\mathrm{SNpc}$ neuronal death. It was previously shown that MPTP neurotoxicity in mice results in an increase in striatal 3nitrotyrosine (a product of $\mathrm{NO}$ and superoxide), which can be attenuated by the administration of 7-NI [30]. In addition, 7-NI can significantly prevent MPTP-related neurotoxicity, as evidenced by the greater number of tyrosine-hydroxylaseimmunostained neurons in 7-NI-pretreated mice [15]. Moreover, this protective effect occurred in a dose-dependent manner, indicating that MPTP-induced toxicity is directly proportional to nNOS activity. Further evidence comes from the observation that nNOS-deficient mice are twofold less affected by MPTP than wild-type and heterozygous mice [31]. Together these findings suggest that nNOS-derived NO plays a critical role in the neurotoxicity of MPTP. Although previous studies also suggested that inducible nitric oxide synthase (iNOS) activity was increased after MPTP exposure [31, 32]; due to the relative selectivity of 7-NI, we thus concluded that the transient cerebral hyperperfusion as showed in our study was a result of increased nNOS activity rather than the iNOS.

FAIR is recognized as a completely noninvasive means of visualizing tissue perfusion. Secondary to its noninvasiveness, this technique allows multiple repeated measurements of $\mathrm{CBF}$ at sufficiently high temporal and spatial resolutions. Our results obtained using FAIR-phMRI are the first to provide in vivo evidence of increased nNOS activity in acute MPTP-induced neurotoxicity. Although FAIR revealed remarkable changes in CBF following MPTP administration, this alteration was not accompanied by metabolic or structural lesions, as evaluated by MRS and DWI. This suggests that FAIR remains a superior tool for detecting early changes in MPTP-induced neurotoxicity.

Apparent diffusion coefficient (ADC) is a DWI-derived quantitative parameter that reflects the degree of tissue water diffusivity restriction. The reduction of ADC has been related to various biological conditions, particularly in the processes that involve cytotoxic edema or increased cellularity (such as inflammation). It has been reported [33] that there was no significant difference of regional ADC values in various brain regions between the PD patients and control group. This is consistent with the result in the present study of rodent PD model. On the other hand, in vivo ${ }^{1} \mathrm{H}$-MRS detects low concentration neuronal metabolites to provide surrogate markers for neuronal damage. Previous in vivo ${ }^{1} \mathrm{H}-\mathrm{MRS}$ studies [3436] showed decreased $\mathrm{N}$-acetylaspartate/creatinine ratio in the lentiform nuclei and striatum of PD patients. However, in the present study, in vivo ${ }^{1} \mathrm{H}$-MRS revealed no significant signal change in rat brain after acute MPTP exposure; only CBF change was observed. It could be largely due to the fact that $\mathrm{NO}$ is an obligatory regulator of cerebral hemodynamics, where CBF is highly sensitive to the alterations of NO level. Whereas under current dosing regimen, the produced NO level may not be sufficiently high to cause neuronal damage that can be detected by ${ }^{1} \mathrm{H}$-MRS.
The increase in CBF revealed in our study is probably due to an NO-cyclic guanosine monophosphate (cGMP)mediated vasodilatory effect $[19,37]$, which might be independent of the toxicogenesis of MPTP. However, given the strong oxidative power of NO, it is likely that it is at least partly involved in the neurotoxicity of MPTP. The main aim of this study was to demonstrate the spatial-temporal distribution of NO in MPTP-induced toxicity. We believe that our findings will facilitate future studies on the role of NO.

Perfusion neuroimaging studies, either by single-photon emission computed tomography [38] or MRI [39], have generally confirmed the presence of a hypoperfusion state in the gray matter of PD patients. These studies have demonstrated that several cerebral regions, including the posterior parietooccipital cortex, precuneus, cuneus, and middle frontal gyri, experience decreases in regional perfusion. Paradoxically, the present study revealed a transient hyperperfusion in the cerebral cortex and striatum in the MPTP animal model, which may be related to overproduction of NO. This finding suggests that a similar hyperperfusion state is present in human PD, and this may represent an early neuroinflammation in the brain. Further research should be conducted to examine the existence of this early hemodynamic alteration in human PD.

We found that MPTP injection caused a persistent elevation in regional CBF in the striatum, suggesting that this brain area is a major source of the neurotoxic NO. Consistent with this notion, the striatum contains a rich density of nNOSpositive neurons and fibers [40]. In contrast, there is no evidence for nNOS immunoreactivity in neurons or fibers in the vicinity of the SNpc [40]. Hypothetically, dopamine nerve terminals in the striatum become the primary target for NO, followed by a secondary retrodegeneration of dopamine cell bodies in the SNpc [41]. Consistent with this hypothesis is the observation that $\mathrm{MPP}^{+}$accumulates primarily in the striatal dopamine terminals, but not in SNpc DAergic neuronal cell bodies [42].

While the cerebral cortex exhibits less nNOS activity, there is one possible explanation for the more-prominent increase in the MPTP-induced CBF increase in this region: in addition to the NO-cGMP-mediated direct relaxation of the vascular smooth muscle, the vasodilation effect of NO may also arise from its counteraction to endogenous vasoconstrictors [20]. The observed change in CBF is hence a complex interplay between $\mathrm{NO}$ and other vasoactive substances (e.g., angiotensin). Therefore, variations in CBF increases across different brain regions do not necessarily reflect the proportional nNOS activities, since it is likely that there are distinct basal regulation mechanisms in these regions.

It is known that NO plays an important role in the normal regulation of cerebral vascular tone [19]. Our findings also show that 7-NI attenuated the basal cortical CBF prior to MPTP administration. This is consistent with previous reports that 7-NI injection results in a decrease in local CBF in the rat brain $[43,44]$. It is worth noting that basal striatal CBF was unaffected by 7-NI injection in the present study, which might have been due to the much lower dosage of 7-NI used.

A major drawback of the present study is the lack of perfusion measurements in the SNpc, which is thought to be a site of PD lesions. This was mainly due to technical 
limitations associated with the use of the single-slice FAIR technique in this work. Multislice FAIR [45] could be implemented to include measurement of SNpc by increasing the slab thickness of slice-selective inversion such that several slices were contained within it. However, multislice FAIR imaging presents two major problems: (1) the integrity of selective inversion across all slices is questionable (i.e., the imperfect inversion pulse profile across slices causes significant errors) [46], and (2) the multislice FAIR approach introduces an increased transit time delay for those slices farther from the edge of the inversion slice [47]. Together, these limitations hinder accurate measurements using multislice FAIR. Nevertheless, the present results warrant further study of the hemodynamics of the SNpc in acute MPTP toxicity.

It has been reported that rats are less susceptible to systemic MPTP toxicity than mice and primates [48], which might be due to systemic MPTP being extensively metabolized by MAO-B in the rat blood-brain barrier, thereby converting MPTP into $\mathrm{MPP}^{+}[49]$. $\mathrm{MPP}^{+}$is a polar molecule that does not readily cross biological membranes, hence preventing it from reaching sites of injury in sufficient concentrations. This view is supported by the direct infusion of MPTP into the rat SNpc causing a selective 50-70\% loss of DAergic neurons, without affecting other neurons or glia at the injection site [50]. Therefore, the differences in susceptibility between species probably arise from their distinct pharmacokinetic profiles. Such differences may have a relatively minimal impact on pharmacodynamic investigations, as in the present research.

As discussed above, several previous studies have shown that 7-NI reduces MPTP-induced neurotoxicity in several animal models, presumably through the inhibition of nNOS. However, Castagnoli et al. demonstrated that 7-NI can also inhibit the MAO-B-catalyzed oxidation of MPTP to $\mathrm{MPP}^{+}$ [51]. In sharp contrast, Schulz et al. reported no effect of 7NI on MAO-B activity [30]. Hence, the exact mechanism underlying the neuroprotective effect of 7-NI against MPTP toxicity remains to be established. Recent data from an in vitro study suggested that 7-NI has only a mild MAO-B-inhibitory effect [52]. It is unlikely that such inhibition could affect the interpretation of the results in the present study.

\section{Conclusion}

In summary, this study has demonstrated that systemic administration of MPTP leads to prominent changes in CBF in striatal and cortical regions of the rodent CNS. Such increases can be prevented by pretreatment with the selective nNOS inhibitor, 7-NI. Thus, our results provide the first in vivo evidence of $\mathrm{NO}$ production in the acute neurotoxicity of MPTP. Given the similarity between the MPTP model and human parkinsonism, this cascade of events may also occur in PD.

\section{Abbreviations}

7-NI: 7-Nitroindazole

ADC: Apparent diffusion coefficient

ATP: Adenosine triphosphate

\begin{tabular}{|c|c|}
\hline CBF: & Cerebral blood flow \\
\hline cGMP: & Cyclic guanosine monophosphate \\
\hline CNS: & Central nervous system \\
\hline DAergic: & Dopaminergic \\
\hline DWI: & Diffusion-weighted imaging \\
\hline FAIR: & Flow-sensitive alternating inversion recovery \\
\hline FOV: & Field of view \\
\hline i.p.: & Intraperitoneally \\
\hline IR-FSE: & Inversion recovery fast spin-echo \\
\hline i.v.: & Intravenous \\
\hline MAO: & Monoamine oxidase \\
\hline $\mathrm{MPP}^{+}:$ & 1-Methyl-4-phenylpyridinium ion \\
\hline MPTP: & $\begin{array}{l}\text { 1-Methyl-4-phenyl-1,2,3,6- } \\
\text { tetrahydropyridine }\end{array}$ \\
\hline MRI: & Magnetic resonance imaging \\
\hline MRS: & Magnetic resonance spectroscopy \\
\hline iNOS: & Inducible nitric oxide synthase \\
\hline nNOS: & Neuronal nitric oxide synthase \\
\hline NO: & Nitric oxide \\
\hline nsIR-FSE: & Non-slice-selective IR-FSE \\
\hline PD: & Parkinson's disease \\
\hline phMRI: & Pharmacological MRI \\
\hline PRESS: & Point-resolved spectroscopy \\
\hline RF: & Radiofrequency \\
\hline ROI: & Region-of-interest \\
\hline SD: & Standard deviation \\
\hline SNpc: & Substantia nigra pars compacta \\
\hline ssIR-FSE: & Slice-selective IR-FSE \\
\hline TE: & Echo time \\
\hline TI: & Inversion time \\
\hline TR: & Repetition time. \\
\hline
\end{tabular}

\section{Conflict of Interests}

The authors declare no conflict financial of interests.

\section{Acknowledgments}

The authors acknowledge technical support from the Functional and Micro-Magnetic Resonance Imaging Center supported by the National Research Program for Genomic Medicine, National Science Council, Taiwan, ROC. The authors thank Mr. Y. L. Pan for providing technological support of MRI.

\section{References}

[1] J. W. Langston, I. Irwin, E. B. Langston, and L. S. Forno, "1Methyl-4-phenylpyridinium ion (MPP+): identification of a metabolite of MPTP, a toxin selective to the substantia nigra," Neuroscience Letters, vol. 48, no. 1, pp. 87-92, 1984.

[2] J. A. Javitch and S. H. Snyder, "Uptake of MPP(+) by dopamine neurons explains selectivity of Parkinsonism-inducing neurotoxin, MPTP," European Journal of Pharmacology, vol. 106, no. 2, pp. 455-456, 1984.

[3] V. G. Desai, R. J. Feuers, R. W. Hart, and S. F. Ali, "MPP+induced neurotoxicity in mouse is age-dependent: evidenced by the selective inhibition of complexes of electron transport," Brain Research, vol. 715, no. 1-2, pp. 1-8, 1996. 
[4] J. B. Schulz, D. R. Henshaw, R. T. Matthews, and M. F. Beal, "Coenzyme Q10 and nicotinamide and a free radical spin trap protect against MPTP neurotoxicity," Experimental Neurology, vol. 132, no. 2, pp. 279-283, 1995.

[5] R. R. Ramsay, J. I. Salach, and T. P. Singer, "Uptake of the neurotoxin 1-methyl-4-phenylpyridine (MPP+) by mitochondria and its relation to the inhibition of the mitochondrial oxidation of NAD+-linked substrates by MPP+," Biochemical and Biophysical Research Communications, vol. 134, no. 2, pp. 743-748, 1986.

[6] R. R. Ramsay, J. Dadgar, A. Trevor, and T. P. Singer, "Energydriven uptake of $\mathrm{N}$-methyl-4-phenylpyridine by brain mitochondria mediates the neurotoxicity of MPTP," Life Sciences, vol. 39, no. 7, pp. 581-588, 1986.

[7] W. J. Nicklas, S. K. Youngster, M. V. Kindt, and R. E. Heikkila, "MPTP, MPP+ and mitochondrial function," Life Sciences, vol. 40, no. 8, pp. 721-729, 1987.

[8] Y. Mizuno, N. Sone, and T. Saitoh, "Effects of 1-methyl-4-phenyl-1,2,3,6-tetrahydropyridine and 1-methyl-4-phenylpyridinium ion on activities of the enzymes in the electron transport system in mouse brain," Journal of Neurochemistry, vol. 48, no. 6, pp. 1787-1793, 1987.

[9] G. P. Davey and J. B. Clark, “Threshold effects and control of oxidative phosphorylation in nonsynaptic rat brain mitochondria," Journal of Neurochemistry, vol. 66, no. 4, pp. 1617-1624, 1996.

[10] P. Chan, L. E. DeLanney, I. Irwin, J. W. Langston, and D. di Monte, "Rapid ATP loss caused by 1-methyl-4-phenyl-1,2,3,6tetrahydropyridine in mouse brain," Journal of Neurochemistry, vol. 57, no. 1, pp. 348-351, 1991.

[11] J. B. Schulz, R. T. Matthews, B. G. Jenkins et al., "Blockade of neuronal nitric oxide synthase protects against excitotoxicity in vivo," Journal of Neuroscience, vol. 15, no. 12, pp. 8419-8429, 1995.

[12] J. B. Schulz, R. T. Matthews, T. Klockgether, J. Dichgans, and M. F. Beal, "The role of mitochondrial dysfunction and neuronal nitric oxide in animal models of neurodegenerative diseases," Molecular and Cellular Biochemistry, vol. 174, no. 1-2, pp. 193197, 1997.

[13] J. Ara, S. Przedborski, A. B. Naini et al., "Inactivation of tyrosine hydroxylase by nitration following exposure to peroxynitrite and 1-methyl-4-phenyl-1,2,3,6-tetrahydropyridine (MPTP)," Proceedings of the National Academy of Sciences of the United States of America, vol. 95, no. 13, pp. 7659-7663, 1998.

[14] T. Spencer Smith, R. H. Swerdlow, W. Davis Parker Jr., and J. P. Bennett Jr., "Reduction of MPP+-induced hydroxyl radica formation and nigrostriatal MPTP toxicity by inhibiting nitric oxide synthase," NeuroReport, vol. 5, no. 18, pp. 2598-2600, 1994.

[15] S. Przedborski, V. Jackson-Lewis, R. Yokoyama, T. Shibata, V. L. Dawson, and T. M. Dawson, "Role of neuronal nitric oxide in 1methyl-4-phenyl-1,2,3,6-tetrahydropyridine (MPTP)-induced dopaminergic neurotoxicity," Proceedings of the National Academy of Sciences of the United States of America, vol. 93, no. 10, pp. 4565-4571, 1996.

[16] J. Garthwaite, S. L. Charles, and R. Chess-Williams, "Endothelium-derived relaxing factor release on activation of NMDA receptors suggests role as intercellular messenger in the brain," Nature, vol. 336, no. 6197, pp. 385-388, 1988.

[17] N. Hogg, V. M. Darley-Usmar, M. T. Wilson, and S. Moncada, "Production of hydroxyl radicals from the simultaneous generation of superoxide and nitric oxide," Biochemical Journal, vol. 281, no. 2, pp. 419-424, 1992.
[18] T. S. Hakim, K. Sugimori, E. M. Camporesi, and G. Andersen, "Half-life of nitric oxide in aqueous solutions with and without haemoglobin," Physiological Measurement, vol. 17, no. 4, pp. 267277, 1996.

[19] S. Moncada, R. M. J. Palmer, and E. A. Higgs, "Nitric oxide: physiology, pathophysiology, and pharmacology," Pharmacological Reviews, vol. 43, no. 2, pp. 109-142, 1991.

[20] V. Bauer and R. Sotníková, "Nitric oxide: the endotheliumderived relaxing factor and its role in endothelial functions," General Physiology and Biophysics, vol. 29, no. 4, pp. 319-340, 2010.

[21] S.-G. Kim, "Quantification of relative cerebral blood flow change by flow-sensitive alternating inversion recovery (FAIR) technique: application to functional mapping," Magnetic Resonance in Medicine, vol. 34, no. 3, pp. 293-301, 1995.

[22] R. A. Leslie and M. F. James, "Pharmacological magnetic resonance imaging: a new application for functional MRI," Trends in Pharmacological Sciences, vol. 21, no. 8, pp. 314-318, 2000.

[23] N. Easton, F. H. Marshall, C. A. Marsden, and K. C. F. Fone, "Mapping the central effects of methylphenidate in the rat using pharmacological MRI BOLD contrast," Neuropharmacology, vol. 57, no. 7-8, pp. 653-664, 2009.

[24] C. Martin and N. R. Sibson, "Pharmacological MRI in animal models: a useful tool for 5-HT research?” Neuropharmacology, vol. 55, no. 6, pp. 1038-1047, 2008.

[25] G. J. Southan and C. Szabó, "Selective pharmacological inhibition of distinct nitric oxide synthase isoforms," Biochemical Pharmacology, vol. 51, no. 4, pp. 383-394, 1996.

[26] W. T. Lee, H. S. Yin, and Y. Z. Shen, "The mechanisms of neuronal death produced by mitochondrial toxin 3-nitropropionic acid: the roles of $\mathrm{N}$-methyl-D-aspartate glutamate receptors and mitochondrial calcium overload," Neuroscience, vol. 112, no. 3, pp. 707-716, 2002.

[27] G. M. Mackenzie, M. J. Jackson, P. Jenner, and C. D. Marsden, "Nitric oxide synthase inhibition and MPTP-induced toxicity in the common marmoset," Synapse, vol. 26, pp. 301-316, 1997.

[28] P. Herscovitch and M. E. Raichle, "What is the correct value for the brain-blood partition coefficient for water?" Journal of Cerebral Blood Flow and Metabolism, vol. 5, no. 1, pp. 65-69, 1985.

[29] N. V. Tsekos, F. Zhang, H. Merkle, M. Nagayama, C. Iadecola, and S.-G. Kim, "Quantitative measurements of cerebral blood flow in rats using the FAIR technique: correlation with previous iodoantipyrine autoradiographic studies," Magnetic Resonance in Medicine, vol. 39, no. 4, pp. 564-573, 1998.

[30] J. B. Schulz, R. T. Matthews, M. M. K. Muqit, S. E. Browne, and M. F. Beal, "Inhibition of neuronal nitric oxide synthase by 7 nitroindazole protects against MPTP-induced neurotoxicity in mice," Journal of Neurochemistry, vol. 64, no. 2, pp. 936-939, 1995.

[31] T. Dehmer, J. Lindenau, S. Haid, J. Dichgans, and J. B. Schulz, "Deficiency of inducible nitric oxide synthase protects against MPTP toxicity in vivo," Journal of Neurochemistry, vol. 74, no. 5, pp. 2213-2216, 2000.

[32] G. T. Liberatore, V. Jackson-Lewis, S. Vukosavic et al., "Inducible nitric oxide synthase stimulates dopaminergic neurodegeneration in the MPTP model of Parkinson disease," Nature Medicine, vol. 5, no. 12, pp. 1403-1409, 1999.

[33] B. Degirmenci, M. Yaman, A. Haktanir, R. Albayrak, M. Acar, and G. Caliskan, "The effects of levodopa use on diffusion coefficients in various brain regions in Parkinson's disease," Neuroscience Letters, vol. 416, no. 3, pp. 294-298, 2007. 
[34] F. Federico, I. L. Simone, V. Lucivero et al., "Proton magnetic resonance spectroscopy in Parkinson's disease and atypical parkinsonian disorders," Movement Disorders, vol. 12, no. 6, pp. 903-909, 1997.

[35] F. Federico, I. L. Simone, V. Lucivero et al., "Proton magnetic resonance spectroscopy in Parkinson's disease and progressive supranuclear palsy," Journal of Neurology Neurosurgery and Psychiatry, vol. 62, no. 3, pp. 239-242, 1997.

[36] C. E. Clarke and M. Lowry, "Systematic review of proton magnetic resonance spectroscopy of the striatum in parkinsonian syndromes," European Journal of Neurology, vol. 8, no. 6, pp. 573-577, 2001.

[37] F. Murad, "Cyclic guanosine monophosphate as a mediator of vasodilation," Journal of Clinical Investigation, vol. 78, no. 1, pp. $1-5,1986$.

[38] J.-L. Hsu, T.-P. Jung, C.-Y. Hsu et al., "Regional CBF changes in Parkinson's disease: a correlation with motor dysfunction," European Journal of Nuclear Medicine and Molecular Imaging, vol. 34, no. 9, pp. 1458-1466, 2007.

[39] T. R. Melzer, R. Watts, M. R. MacAskill et al., "Arterial spin labelling reveals an abnormal cerebral perfusion pattern in Parkinson's disease," Brain, vol. 134, no. 3, pp. 845-855, 2011.

[40] D. S. Bredt, C. E. Glatt, P. M. Hwang, M. Fotuhi, T. M. Dawson, and S. H. Snyder, "Nitric oxide synthase protein and mRNA are discretely localized in neuronal populations of the mammalian CNS together with NADPH diaphorase," Neuron, vol. 7, no. 4, pp. 615-624, 1991.

[41] S. Przedborski, M. Levivier, H. Jiang et al., "Dose-dependent lesions of the dopaminergic nigrostriatal pathway induced by intrastriatal injection of 6-hydroxydopamine," Neuroscience, vol. 67 , no. 3, pp. 631-647, 1995.

[42] M. Herkenham, M. D. Little, K. Bankiewicz, S.-C. Yang, S. P. Markey, and J. N. Johannessen, "Selective retention of MPP+ within the monoaminergic systems of the primate brain following MPTP administration: an in vivo autoradiographic study," Neuroscience, vol. 40, no. 1, pp. 133-158, 1991.

[43] K. Tanaka, Y. Fukuuchi, S. Gomi et al., "Inhibition of nitric oxide synthesis impaires autoregulation of local cerebral blood flow in the rat," NeuroReport, vol. 4, no. 3, pp. 267-270, 1993.

[44] P. A. T. Kelly, I. M. Ritchie, and G. W. Arbuthnott, "Inhibition of neuronal nitric oxide synthase by 7-nitroindazole: effects upon local cerebral blood flow and glucose use in the rat," Journal of Cerebral Blood Flow and Metabolism, vol. 15, no. 5, pp. 766-773, 1995.

[45] S.-G. Kim, N. V. Tsekos, and J. Ashe, "Multi-slice perfusionbased functional MRI using the FAIR technique: comparison of CBF and BOLD effects," NMR in Biomedicine, vol. 10, no. 4-5, pp. 191-196, 1997.

[46] L. R. Frank, E. C. Wong, and R. B. Buxton, "Slice profile effects in adiabatic inversion: application to multislice perfusion imaging," Magnetic Resonance in Medicine, vol. 38, no. 4, pp. 558564, 1997.

[47] F. Calamante, D. L. Thomas, G. S. Pell, J. Wiersma, and R. Turner, "Measuring cerebral blood flow using magnetic resonance imaging techniques," Journal of Cerebral Blood Flow and Metabolism, vol. 19, no. 7, pp. 701-735, 1999.

[48] A. Giovanni, B.-A. Sieber, R. E. Heikkila, and P. K. Sonsalla, "Studies on species sensitivity to the dopaminergic neurotoxin 1-methyl-4- phenyl-1,2,3,6-tetrahydropyridine. Part 1: systemic administration," Journal of Pharmacology and Experimental Therapeutics, vol. 270, no. 3, pp. 1000-1007, 1994.
[49] R. N. Kalaria, M. J. Mitchell, and S. I. Harik, "Correlation of 1methyl-4-phenyl-1,2,3,6-tetrahydropyridine neurotoxicity with blood-brain barrier monoamine oxidase activity," Proceedings of the National Academy of Sciences of the United States of America, vol. 84, no. 10, pp. 3521-3525, 1987.

[50] L. M. Sayre, P. K. Arora, L. A. Iacofano, and S. I. Harik, "Comparative toxicity of MPTP, MPP+ and 3,3-dimethyl-MPDP+ to dopaminergic neurons of the rat substantia nigra," European Journal of Pharmacology, vol. 124, no. 1-2, pp. 171-174, 1986.

[51] K. Castagnoli, S. Palmer, A. Anderson, T. Bueters, and N. Castagnoli Jr., "The neuronal nitric oxide synthase inhibitor 7nitroindazole also inhibits the monoamine oxidase-B-catalyzed oxidation of 1 -methyl-4-phenyl- 1,2,3,6-tetrahydropyridine," Chemical Research in Toxicology, vol. 10, no. 4, pp. 364-368, 1997.

[52] B. Thomas, K. S. Saravanan, and K. P. Mohanakumar, "In vitro and in vivo evidences that antioxidant action contributes to the neuroprotective effects of the neuronal nitric oxide synthase and monoamine oxidase-B inhibitor, 7-nitroindazole," Neurochemistry International, vol. 52, no. 6, pp. 990-1001, 2008. 


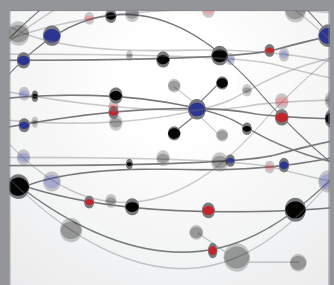

The Scientific World Journal
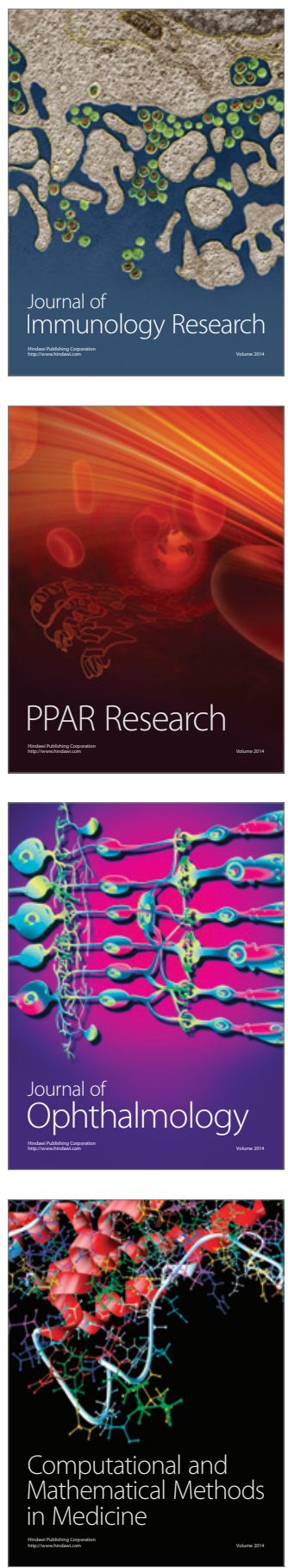

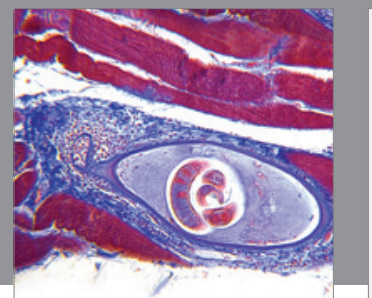

Gastroenterology

Research and Practice
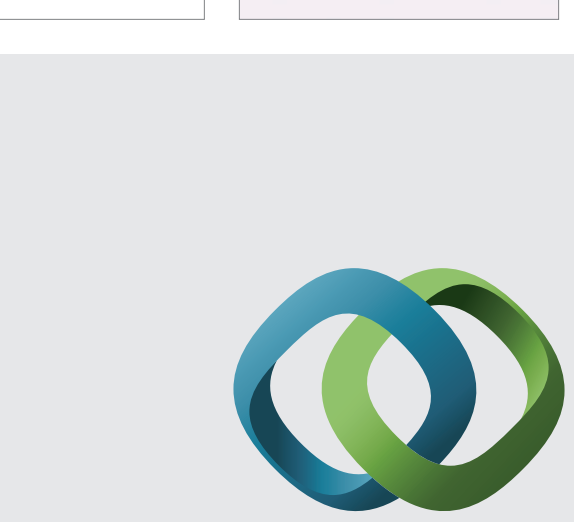

\section{Hindawi}

Submit your manuscripts at

http://www.hindawi.com
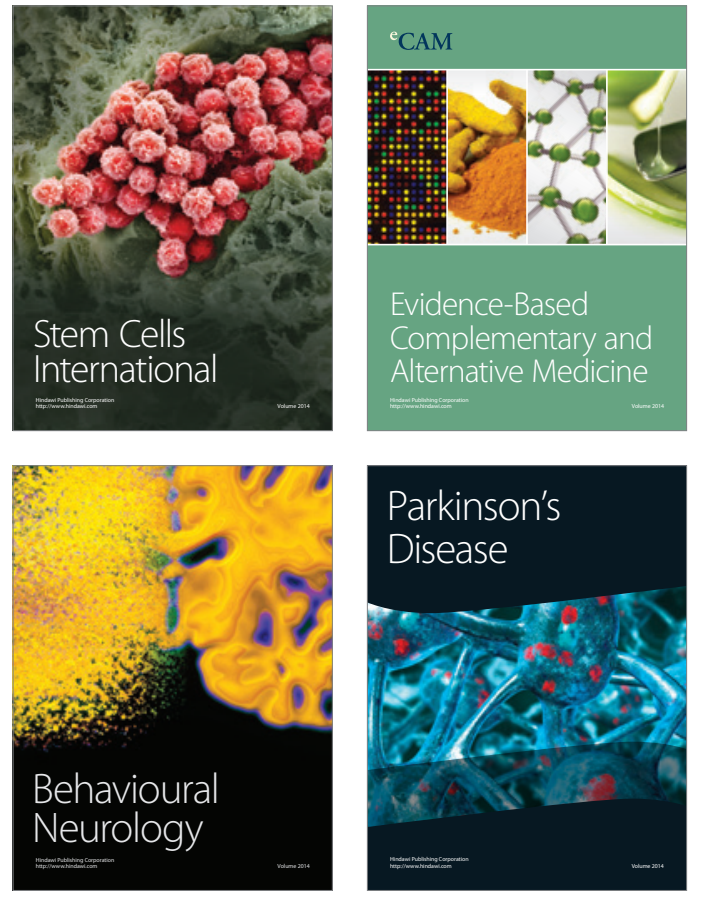
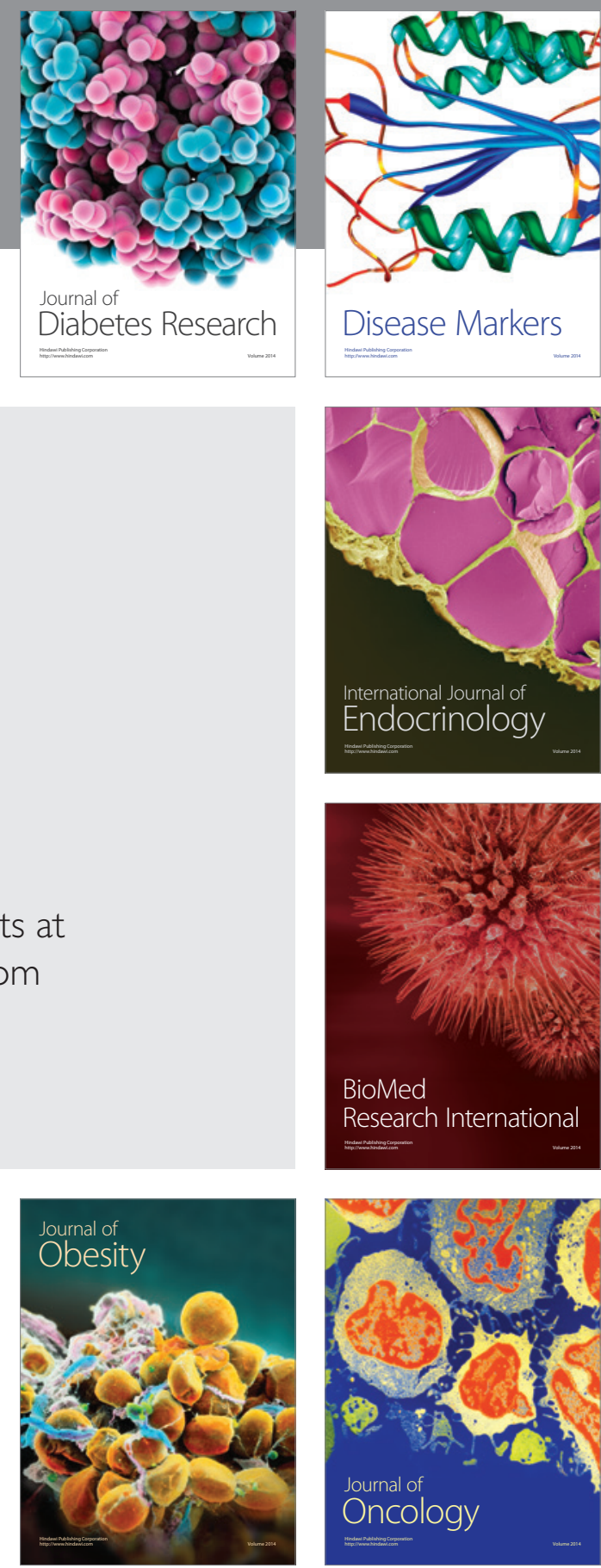

Disease Markers
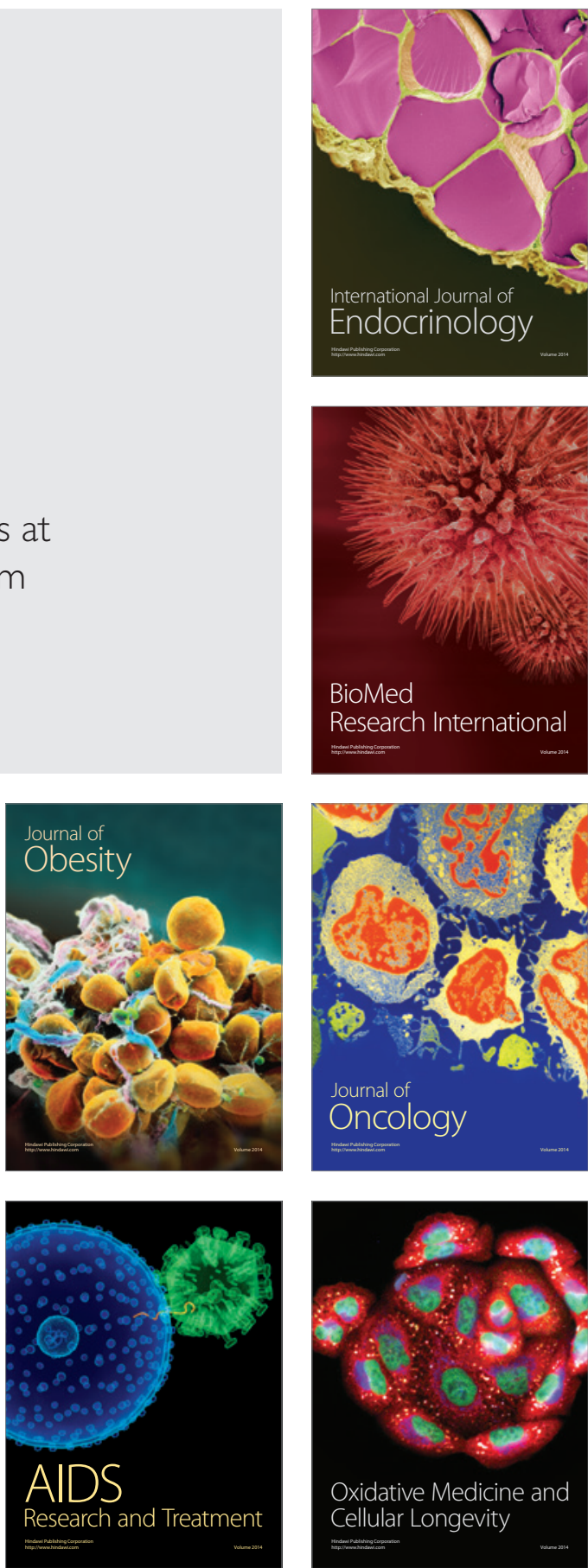